\title{
Supplementary Document
}

\section{Tungsten Suboxide Nanoneedles as Effective Thermal Shield Through}

\section{Near-infrared Reflection and Absorption}

Jiaxin Yan ${ }^{1}$, Ashutosh Rath ${ }^{1,2}$, Hongyu Wang ${ }^{1}$, Ng Zhen Quan, Cavin ${ }^{1,3}$, Stephen J. Pennycook ${ }^{1}$ and Daniel H. C. Chua ${ }^{1 *}$

${ }^{1}$ Department of Materials Science and Engineering, National University of Singapore, 117575, Singapore

${ }^{2}$ CSIR-Institute of Minerals and Materials Technology, Bhubaneswar, Odisha, 751013, India

${ }^{3}$ NUS Graduate School for Integrative Sciences and Engineering, National University of Singapore, 119077, Singapore

Number of pages: 5

Number of figures: 2

Number of tables: 1

\section{Dimensions of Nanoneedles with Different Morphology}

Table $\mathbf{S 1}$ is a summary of the length and thickness dimensions of the nanoneedles with different dimensions. The measurement is done through processing the SEM images with ImageJ software. The numbers in this table are taken through averaging the readings from 10 measurements.

\begin{tabular}{|l|l|l|l|l|}
\hline & Forest-like & $\begin{array}{l}\text { Nanoneedle } \\
\text { buds }\end{array}$ & $\begin{array}{l}\text { Medium } \\
\text { Nanoneedles }\end{array}$ & $\begin{array}{l}\text { Long } \\
\text { Nanoneedles }\end{array}$ \\
\hline Thickness & $29 \mathrm{~nm}$ & $25 \mathrm{~nm}$ & $26 \mathrm{~nm}$ & $44 \mathrm{~nm}$ \\
\hline Length & $402 \mathrm{~nm}$ & $177 \mathrm{~nm}$ & $432 \mathrm{~nm}$ & $1129 \mathrm{~nm}$ \\
\hline
\end{tabular}

Table S1: Dimensions of Nanoneedles with different morphology (as described in figure 3(a) and figure 4) 


\section{Visualization of Specular NIR Reflection}

Figure S1 provides a direct visualization of specular NIR reflection of our samples using FLIR one thermal camera. Figure S1 (a) is the thermal camera image showing the NIR reflection of the camera itself (figure S1 (b)). That is, the infrared spectrum radiated by the thermal camera is specular reflected by our sample (instead of transmitting through). This, together with figure 8 (c) in the main text, further proves the NIR reflective property of our sample.
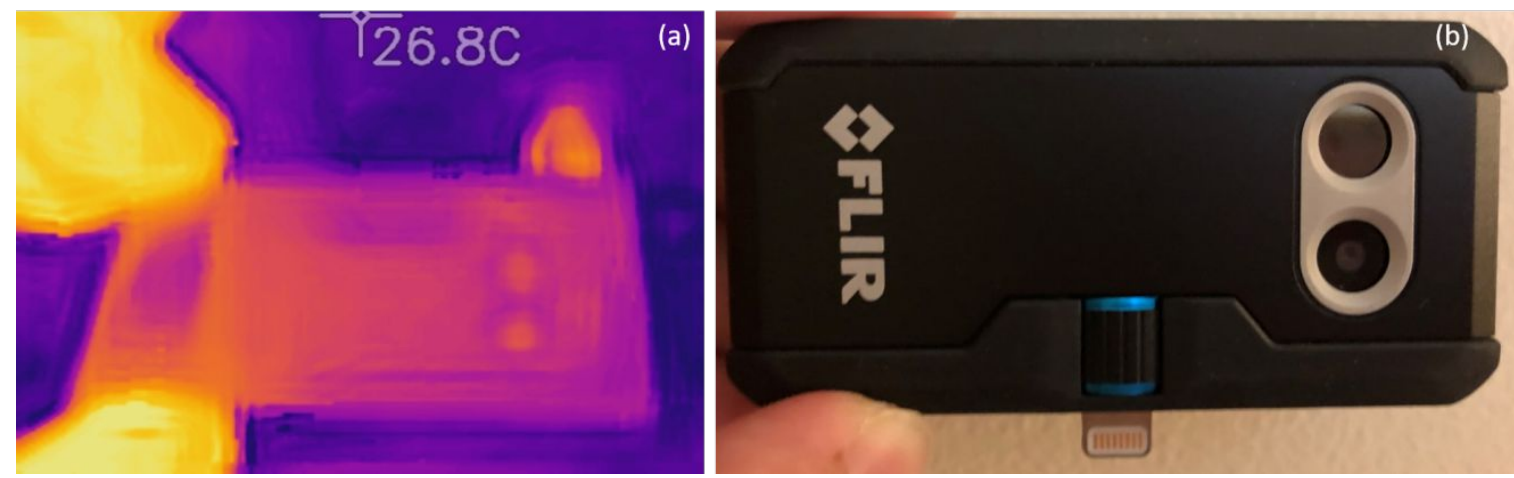

Figure S1 Thermal camera imaging (a) showing the NIR reflection of the camera itself (b), further proving the NIR reflective property of our sample.

\section{Crystallographic Information File (cif) Unit Cell Visualization}

Figure S2 (a), (b), (c) and (d) are the $\mathrm{W}_{17} \mathrm{O}_{47}$ cif unit cell model viewed from [111] direction, $\mathrm{x}$-axis, $\mathrm{y}$-axis and $\mathrm{z}$-axis respectively. The cif file was obtained from crystallography open database ${ }^{1-5}$.

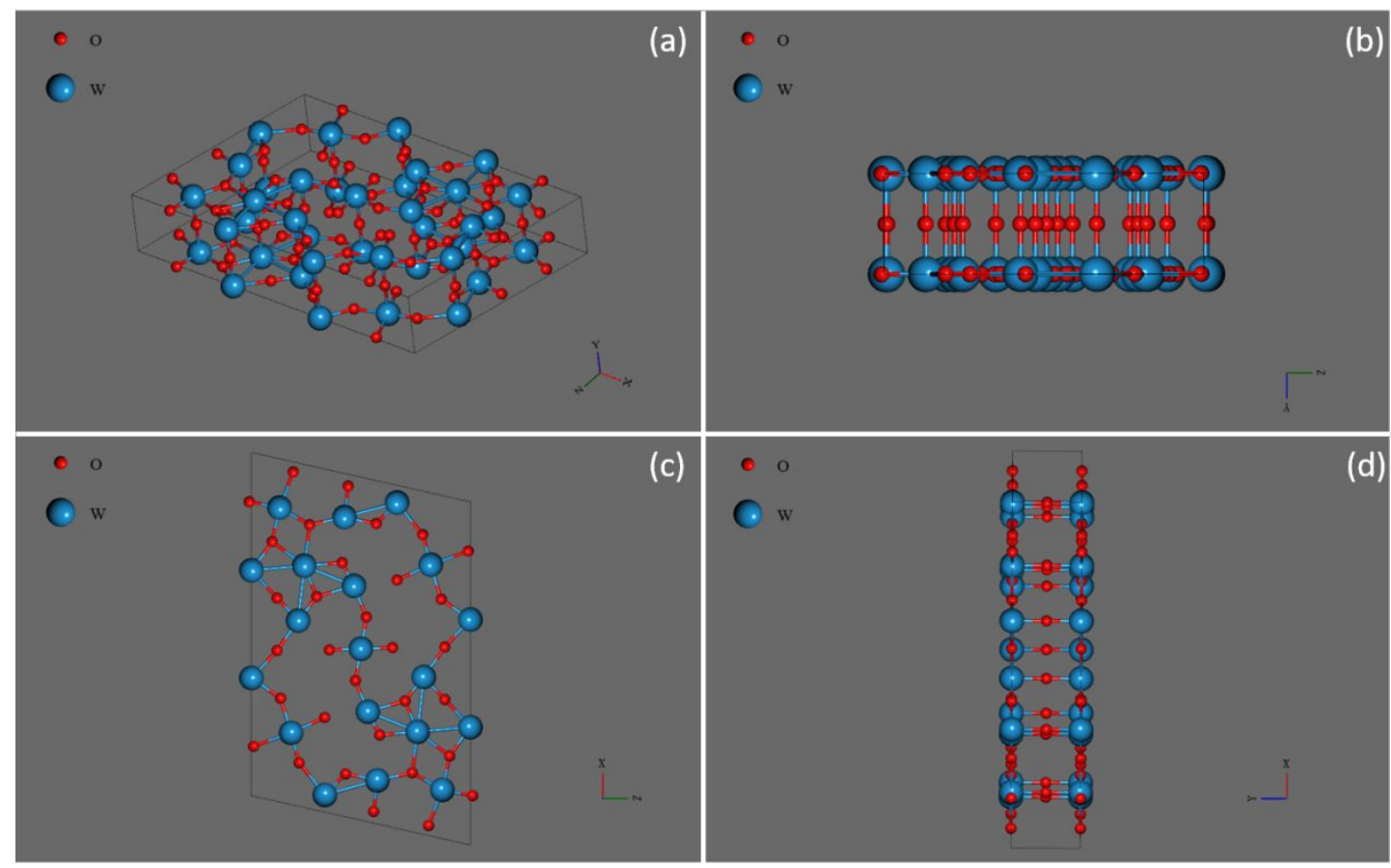

Figure $\mathbf{S 2}$ cif models of $\mathrm{W}_{17} \mathrm{O}_{47}$ unit cell. From (a) to (d), the viewing directions are [111], $\mathrm{x}$-axis, $\mathrm{y}$-axis, and z-axis respectively 


\section{Input file script for Quantum Espresso calculation}

Quantum Espresso relies on a user generated input file, which contains calculation parameters, to run a proper calculation. The following is the input file script used for calculation in this paper.

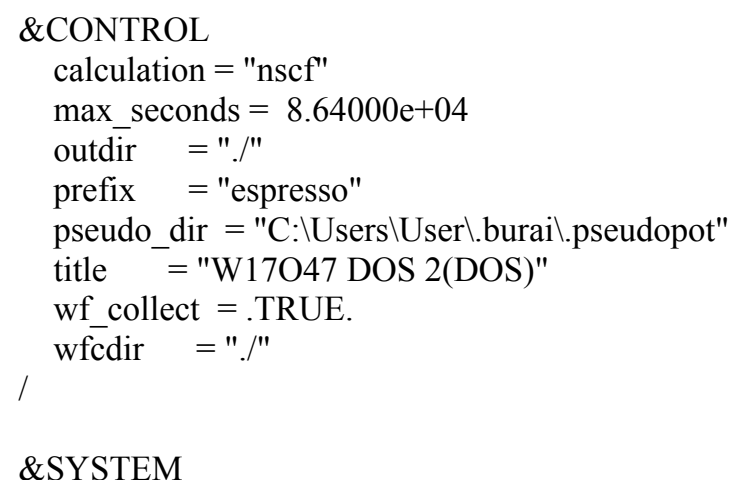




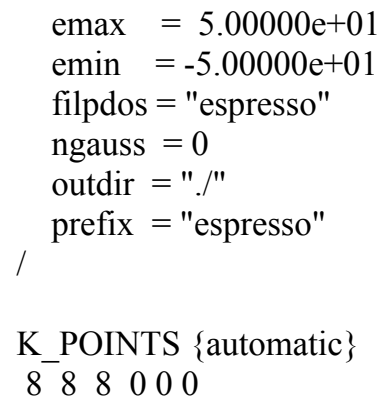




$\begin{array}{lccc}\text { O-2 } & 3.332458 & 0.000000 & 7.049439 \\ \text { W } & 9.616402 & 0.000000 & 2.562339 \\ \text { W } & 6.519193 & 0.000000 & 9.467420 \\ \text { W } & 11.526698 & 0.000000 & 5.629927 \\ \text { W } & 4.608896 & 0.000000 & 6.399832 \\ \text { O-2 } & 14.880148 & 0.000000 & 3.199916 \\ \text { O-2 } & 1.255447 & 0.000000 & 8.829843 \\ \text { O-2 } & 12.630438 & 1.893500 & 2.899172 \\ \text { O-2 } & 3.505156 & 1.893500 & 9.130587 \\ \text { O-2 } & 8.162410 & 0.000000 & 10.622277 \\ \text { O-2 } & 7.973185 & 0.000000 & 1.407482 \\ \text { O-2 } & 15.297948 & 1.893500 & 5.112647 \\ \text { O-2 } & 0.837647 & 1.893500 & 6.917111 \\ \text { O-2 } & 16.099570 & 1.893500 & 7.999789 \\ \text { O-2 } & 0.036024 & 1.893500 & 4.029969 \\ \text { O-2 } & 8.067797 & 1.893500 & 6.014879 \\ \text { W } & 15.818556 & 0.000000 & 1.539809 \\ \text { W } & 0.317039 & 0.000000 & 10.489949 \\ \text { O-2 } & -1.641504 & 0.000000 & 9.732075 \\ \text { O-2 } & 17.777099 & 0.000000 & 2.297684\end{array}$

\section{References}

(1) Quirós Olozábal, M.; Gražulis, S.; Girdzijauskaitė, S.; Merkys, A. Using SMILES strings for the description of chemical connectivity in the Crystallography Open Database. 2018.

(2) Merkys, A.; Vaitkus, A.; Butkus, J.; Okulič-Kazarinas, M.; Kairys, V.; Gražulis, S. COD:: CIF:: Parser: an error-correcting CIF parser for the Perl language. Journal of applied crystallography 2016, 49 (1), 292-301.

(3) Gražulis, S.; Merkys, A.; Vaitkus, A.; Okulič-Kazarinas, M. Computing stoichiometric molecular composition from crystal structures. Journal of applied crystallography 2015, 48 (1), 85-91.

(4) Gražulis, S.; Daškevič, A.; Merkys, A.; Chateigner, D.; Lutterotti, L.; Quiros, M.; Serebryanaya, N. R.; Moeck, P.; Downs, R. T.; Le Bail, A. Crystallography Open Database (COD): an open-access collection of crystal structures and platform for world-wide collaboration. Nucleic acids research 2012, 40 (D1), D420-D427.

(5) Downs, R. T.; Hall-Wallace, M. The American Mineralogist crystal structure database. American Mineralogist 2003, 88 (1), 247-250. 